\title{
Diagnosis of gestational diabetes mellitus: falling through the net
}

\author{
Claire L. Meek ${ }^{1,2,3}$ - Hannah B. Lewis ${ }^{1}$. \\ Charlotte Patient ${ }^{4}$. Helen R. Murphy ${ }^{1,2}$. \\ David Simmons ${ }^{2,5}$
}

Received: 24 February 2015 / Accepted: 8 May 2015 / Published online: 14 June 2015

(C) The Author(s) 2015. This article is published with open access at Springerlink.com

\begin{abstract}
Aims/hypothesis Gestational diabetes mellitus (GDM) is associated with increased risks to mother and child, but globally agreed diagnostic criteria remain elusive. Identification of women with GDM is important, as treatment reduces adverse outcomes such as perinatal death, shoulder dystocia and neonatal hypoglycaemia. Recently, the UK's National Institute for Health and Care Excellence (NICE) recommended new diagnostic thresholds for GDM which are different from the International Association of the Diabetes and Pregnancy Study Groups (IADPSG) criteria endorsed by the WHO. The study aim was to assess neonatal and obstetric outcomes among women who would test positive for the IADPSG criteria but negative for the NICE 2015 criteria.

Methods Data from 25,543 consecutive singleton live births (2004-2008) were obtained retrospectively from hospital records. Women were screened with a random plasma glucose (RPG; $12-16$ weeks) and a $50 \mathrm{~g}$ glucose challenge test (GCT;
\end{abstract}

Claire L. Meek

clm70@cam.ac.uk

1 The Wellcome Trust-MRC Institute of Metabolic Science, Metabolic Research Laboratories, University of Cambridge, Addenbrooke's Hospital, Box 289, Hills Road, Cambridge CB2 0QQ, UK

2 Wolfson Diabetes and Endocrinology Clinic, Cambridge University Hospitals, Addenbrooke's Hospital, Cambridge, UK

3 Department of Clinical Biochemistry, Cambridge University Hospitals, Addenbrooke's Hospital, Cambridge, UK

4 Department of Obstetrics and Gynaecology, Cambridge University Hospitals, The Rosie Hospital, Cambridge, UK

5 School of Medicine, University of Western Sydney, Campbelltown, Sydney, NSW, Australia
26-28 weeks). If RPG $>7.0 \mathrm{mmol} / \mathrm{l}, \mathrm{GCT}>7.7 \mathrm{mmol} / \mathrm{l}$ or symptoms were present, a $75 \mathrm{~g}$ OGTT was offered $(n=3,848)$. Results In this study, GDM prevalence was $4.13 \%$ (NICE 2015 ) and $4.62 \%$ (IADPSG). Women who 'fell through the net', testing NICE-negative but IADPSG-positive $(n=387)$, had a higher risk of having a large-for-gestational-age (LGA) infant (birthweight $>90$ th percentile for gestational age; adjusted OR [95\% CI] 3.12 [2.44, 3.98]), Caesarean delivery $(1.44[1.15,1.81])$ and polyhydramnios $(6.90$ [3.94, 12.08]) compared with women with negative screening results and no OGTT $(n=21,695)$. LGA risk was highest among women with fasting plasma glucose $5.1-5.5 \mathrm{mmol} / 1(n=$ 167): the mean birthweight was $350 \mathrm{~g}$ above that of the reference population and $37.7 \%$ of infants were LGA.

Conclusions/interpretation The IADPSG criteria identify women at substantial risk of complications who would not be identified by the NICE 2015 criteria.

Keywords Diabetes $\cdot$ Diagnosis $\cdot$ Gestational $\cdot$ Macrosomia $\cdot$ Pregnancy $\cdot$ Screening

\begin{tabular}{|c|c|}
\hline \multicolumn{2}{|c|}{ Abbreviations } \\
\hline ACOG & $\begin{array}{l}\text { American College of Obstetricians and } \\
\text { Gynecologists }\end{array}$ \\
\hline CS & Caesarean section \\
\hline FPG & Fasting plasma glucose \\
\hline GDM & Gestational diabetes mellitus \\
\hline GCT & Glucose challenge test \\
\hline IADPSG & $\begin{array}{l}\text { International Association of the Diabetes and } \\
\text { Pregnancy Study Groups }\end{array}$ \\
\hline LGA & Large for gestational age \\
\hline NICE & National Institute for Health and Care Excellence \\
\hline NICU & Neonatal intensive care unit \\
\hline NNT & Number needed to treat \\
\hline
\end{tabular}


RPG Random plasma glucose

SGA Small for gestational age

\section{Introduction}

Gestational diabetes mellitus (GDM), defined as carbohydrate intolerance causing hyperglycaemia with first onset or recognition in pregnancy $[1,2]$, is increasing in incidence in many populations worldwide as obesity becomes more prevalent [3]. Untreated GDM results in poor maternal and fetal outcomes: women with GDM are more likely to suffer preeclampsia, operative delivery and stillbirth [4], and infants are at higher risk of preterm delivery and macrosomia or large for gestational age (LGA), which is associated with birth injury, respiratory distress and neonatal hypoglycaemia [5]. In the longer term, children born to mothers with GDM are at greater risk of obesity and type 2 diabetes in later life, a phenomenon attributed to the effects of intrauterine exposure to hyperglycaemia $[6,7]$.

Fortunately, many of these risks can be reduced by identification of GDM pregnancies and prompt intervention to reduce maternal antenatal hyperglycaemia $[8,9]$. One barrier to case identification has been the lack of a universally accepted set of diagnostic criteria for GDM. The International Association of the Diabetes and Pregnancy Study Groups (IADPSG) proposed diagnostic criteria which were based upon an OR of 1.75 for negative pregnancy outcomes (Table 1) using data from the Hyperglycaemia and Adverse Pregnancy Outcomes (HAPO) study (75 g OGTT $0 \mathrm{~h} \geq 5.1 \mathrm{mmol} / 1,1 \mathrm{~h} \geq 10.0 \mathrm{mmol} /$ $1,2 \mathrm{~h} \geq 8.5 \mathrm{mmol} / \mathrm{l})[10,11]$. However, these criteria used lower fasting plasma glucose (FPG) thresholds than other criteria in common use (Table 1) and added a $1 \mathrm{~h}$ criterion, leading to concerns about increased diagnosis rates, resource allocation and increased medicalisation of pregnancy $[12,13]$. The IADPSG criteria have been adopted by the WHO [2] and the ADA [14] but were not endorsed at the National Institutes of Health summit in the USA [12] nor by the National Institute for Health and Care Excellence (NICE) in the UK due to concerns about treatment costs and the limited evidence of benefit for treating at lower diagnostic thresholds. NICE proposed alternative criteria for adoption in 2015 (75 g OGTT $0 \mathrm{~h}$ $\geq 5.6 \mathrm{mmol} / \mathrm{l} ; 2 \mathrm{~h} \geq 7.8 \mathrm{mmol} / \mathrm{l}$ ) [15].

The aim of the current study was to retrospectively compare outcomes among women who would test positive for the IADPSG criteria for GDM but negative for NICE 2015 diagnostic criteria.

\section{Methods}

Population and standard care Data from all singleton pregnancies (2004-2008) at Cambridge University Hospitals
National Health Service Foundation Trust were obtained retrospectively from hospital medical and obstetric records as part of an approved service evaluation. At that time in our institution all pregnant women were invited to be screened at antenatal booking with a random plasma glucose (RPG; $n=$ 17,736; typically at $12-16$ weeks' gestation). Women with $\mathrm{RPG}>7.0 \mathrm{mmol} / \mathrm{l}$ or a previous diagnosis of GDM were offered a $75 \mathrm{~g}$ OGTT. All women without known GDM/preexisting diabetes were screened at 26-28 weeks with a $50 \mathrm{~g}$ glucose challenge test (GCT). Women with a GCT result $>7.7 \mathrm{mmol} / \mathrm{l}$ were then referred for a $75 \mathrm{~g}$ OGTT [16]. Additional OGTTs were performed in later pregnancy if symptoms were present. Therefore, all women who had an OGTT $(n=3$, 848) had already had at least one abnormal result on glucose testing during pregnancy, symptoms consistent with hyperglycaemia, or GDM in a previous pregnancy. Women with known pre-existing diabetes were excluded from the study. The WHO 1999 criteria were used for GDM diagnosis until August 2007 (75 g OGTT $0 \mathrm{~h} \geq 7.1 \mathrm{mmol} / \mathrm{l} ; 2 \mathrm{~h}$ $\geq 7.8 \mathrm{mmol} / \mathrm{l}$ ) and the modified WHO 1999 criteria thereafter (75 g OGTT $0 \mathrm{~h} \geq 6.1 \mathrm{mmol} / \mathrm{l} ; 2 \mathrm{~h} \geq 7.8 \mathrm{mmol} / \mathrm{l}$; Table 1 ). Following diagnosis, women with GDM were seen every 2 4 weeks at a multidisciplinary clinic, encouraged to monitor their blood glucose levels and offered lifestyle counselling. Women who had evidence of persistent hyperglycaemia were offered treatment with insulin, metformin or both [17]. Women with GDM were offered regular ultrasound scans during pregnancy (at 12, 20, 28 and 36 weeks), whereas non-diabetic women usually have two routine ultrasound scans (at 12 and 20 weeks).

Laboratory analysis Both venous and capillary samples were used during 2004 and 2008 for glucose testing in our institution. Venous blood was collected using fluoride oxalate tubes and analysed using a hexokinase method (Dimension RxL Max Clinical Chemistry System; Siemens Healthcare Diagnostics, Deerfield, IL, USA) in our laboratory accredited by Clinical Pathology Accreditation, UK. Capillary samples were analysed using the Bayer Elite glucose monitoring system (Bayer, Newbury, UK). Although both laboratory and point-of-care methods were regularly calibrated, small differences exist between capillary and venous glucose testing [18]. The same diagnostic criteria were used for both capillary and venous tests.

Definitions Macrosomia was defined as birthweight $>4 \mathrm{~kg}$. LGA was defined as birthweight $>90$ th percentile for gestational age and was calculated for babies born at 24-41 weeks' gestation using the WHO weight percentile calculator with a mean birthweight of 3,542 g (SD $437 \mathrm{~g}$ ) (World Health Organisation Weight percentiles calculator, available from www. who.int/reproductivehealth/topics/best_practices/weight percentiles_calculator.xls; accessed 20 April 2015) [19-21]. 
Pre-eclampsia was defined as systolic blood pressure $\geq 140 \mathrm{mmHg}$ and/or diastolic blood pressure $\geq 90 \mathrm{mmHg}$ on two or more occasions with proteinuria $\geq 1+$ on dipstick. Patients with no blood pressure recorded who were on the preeclampsia treatment pathway before or during labour were also considered to have pre-eclampsia. Patients with chronic hypertension prior to pregnancy were not considered to have pre-eclampsia. Preterm delivery was defined as delivery prior to 37 weeks' gestation. Polyhydramnios was defined as excessive amniotic fluid, corresponding to the deepest vertical pool $\geq 8 \mathrm{~cm}$ on ultrasound or an amniotic fluid index $>95$ th percentile for the corresponding gestational age. Antepartum haemorrhage was defined as any blood loss from the vagina after the 24th week of gestation. Postpartum haemorrhage was defined as blood loss of $>500 \mathrm{ml}$ following delivery, or the requirement for a blood transfusion.

Statistical analysis Data were collected for demographic information, glucose screening results and pregnancy outcomes. Women were classified into groups according to their OGTT results: OGTT not done; NICE-negative IADPSG-negative; NICE-positive IADPSG-positive; NICE-negative IADPSGpositive; and NICE-positive IADPSG-negative (Table 1, Fig. 1). Groups were further divided according to the OGTT criterion that was abnormal. Women could test positive for some but not all GDM diagnostic criteria by having an FPG 5.1-5.5 mmol/1 (IADPSG-only $0 \mathrm{~h}$ group), an OGTT $1 \mathrm{~h}$ glucose $\geq 10.0 \mathrm{mmol} / \mathrm{l}$ (IADPSG-only $1 \mathrm{~h}$ group) or an OGTT $2 \mathrm{~h}$ glucose 7.8-8.4 mmol/l (NICE-only $2 \mathrm{~h}$ group, which was also the NICE-positive IADPSG-negative group) (Table 1, Fig. 1).

Participant characteristics are presented as $n(\%)$ for categorical data and mean $(95 \% \mathrm{CI})$ for continuous data. Differences between each OGTT classification group and the reference population were tested using Fisher's exact test and linear regression, respectively. The reference population was considered to be all women who did not have an OGTT ( $n=$ $21,695)$. These women had a GCT result $<7.8 \mathrm{mmol} / \mathrm{l}$, but some might have had fasting hyperglycaemia that would not have been identified using the GCT.

Associations between GDM classification group and maternal or fetal outcomes compared with the reference population were estimated using logistic regression, and results are presented as ORs (95\% CIs). Results are presented as unadjusted models and models which were adjusted for potential confounders [10]. Analyses of LGA, small for gestational age (SGA; birthweight $<10$ th percentile for gestational age) and pre-eclampsia outcomes were adjusted for maternal BMI, maternal age, parity, maternal smoking and ethnicity. These analyses were not adjusted for gestational age at birth, as it was not considered a true confounder for pre-eclampsia, and LGA and SGA already incorporate gestational age within their definitions. Macrosomia, Caesarean section (CS), instrumental delivery, stillbirth, infant admission to the neonatal intensive care unit (NICU), polyhydramnios, ante- or postpartum haemorrhage, and 1 or 5 min Apgar scores were adjusted for maternal BMI, maternal age, parity, maternal smoking, ethnicity and estimated gestational age at birth. The preterm delivery outcome was adjusted for maternal BMI, maternal age, parity, maternal smoking, ethnicity, pre-eclampsia and antepartum haemorrhage. To make allowance for multiple testing, a significance level of $p \leq 0.001$ was considered significant and $p \leq 0.01$ was considered a trend. Statistical analysis was performed using Stata version 12.0 software (StataCorp LP, College Station, TX, USA).

\section{Results}

Records were obtained for 25,789 births; 25,543 records were included in the analysis after exclusion of pregnancies resulting in miscarriage $(n=59)$ or termination $(n=65)$, those with no birthweight information $(n=3)$, duplicate data $(n=20)$ and records consistent with overt diabetes $(\mathrm{RPG} \geq 11.1 \mathrm{mmol} / 1$ at booking; $n=99)$. Over $99.9 \%$ of records had data available for pregnancy outcome, mode of delivery and antenatal complications; $84.9 \%$ of records had data available for their usual maternal adult BMI. Characteristics of the study population are described in Table 2.

A total of 3,848 (15.1\%) antenatal OGTTs were performed, of which 2,406 (62.5\%) were negative for GDM according to both IADPSG and the proposed NICE 2015 criteria, and 794 women $(20.6 \%)$ had GDM according to both IADPSG and NICE 2015 criteria. In this study, the prevalence of GDM was

Table 1 Current and recent criteria used for diagnosis of GDM based on the OGTT

\begin{tabular}{|c|c|c|c|c|c|}
\hline OGTT criterion & $\begin{array}{l}\text { IADPSG, WHO } 2013 \\
\text { and ADA } 2014 \text { [11] }\end{array}$ & WHO 1999 & Modified WHO 1999 & Proposed NICE 2015 & ACOG \\
\hline Diagnostic requirements & $\begin{array}{l}\text { One abnormality } \\
\text { on } 75 \text { g OGTT }\end{array}$ & $\begin{array}{l}\text { One abnormality } \\
\text { on } 75 \text { g OGTT }\end{array}$ & $\begin{array}{l}\text { One abnormality } \\
\text { on } 75 \mathrm{~g} \text { OGTT }\end{array}$ & $\begin{array}{l}\text { One abnormality } \\
\text { on } 75 \text { g OGTT }\end{array}$ & $\begin{array}{l}\text { Two abnormalities } \\
\text { on } 100 \mathrm{~g} \text { OGTT }\end{array}$ \\
\hline FPG, mmol/l (mg/dl) & $\geq 5.1(\geq 92)$ & $\geq 7.1(\geq 128)$ & $\geq 6.1(\geq 110)$ & $\geq 5.6(\geq 101)$ & $\geq 5.3(\geq 95)$ \\
\hline OGTT 1 h glucose, mmol/l (mg/dl) & $\geq 10.0(\geq 180)$ & - & - & - & $\geq 10.0(\geq 180)$ \\
\hline OGTT 2 h glucose, mmol/l (mg/dl) & $\geq 8.5(\geq 153)$ & $\geq 7.8(\geq 140)$ & $\geq 7.8(\geq 140)$ & $\geq 7.8(\geq 140)$ & $\geq 8.6(\geq 154)$ \\
\hline OGTT 3 h glucose, mmol/l (mg/dl) & - & - & - & - & $\geq 7.8(\geq 140)$ \\
\hline
\end{tabular}


Fig. 1 Classification of pregnant women according to GDM diagnosis. Data are mean $(95 \%$ CI). BW, birthweight; LGA classified as BW $>90$ th percentile for gestational age. ${ }^{\mathrm{a}}$ This group may contain some patients with undiagnosed GDM due to fasting hyperglycaemia that is not identified by the GCT

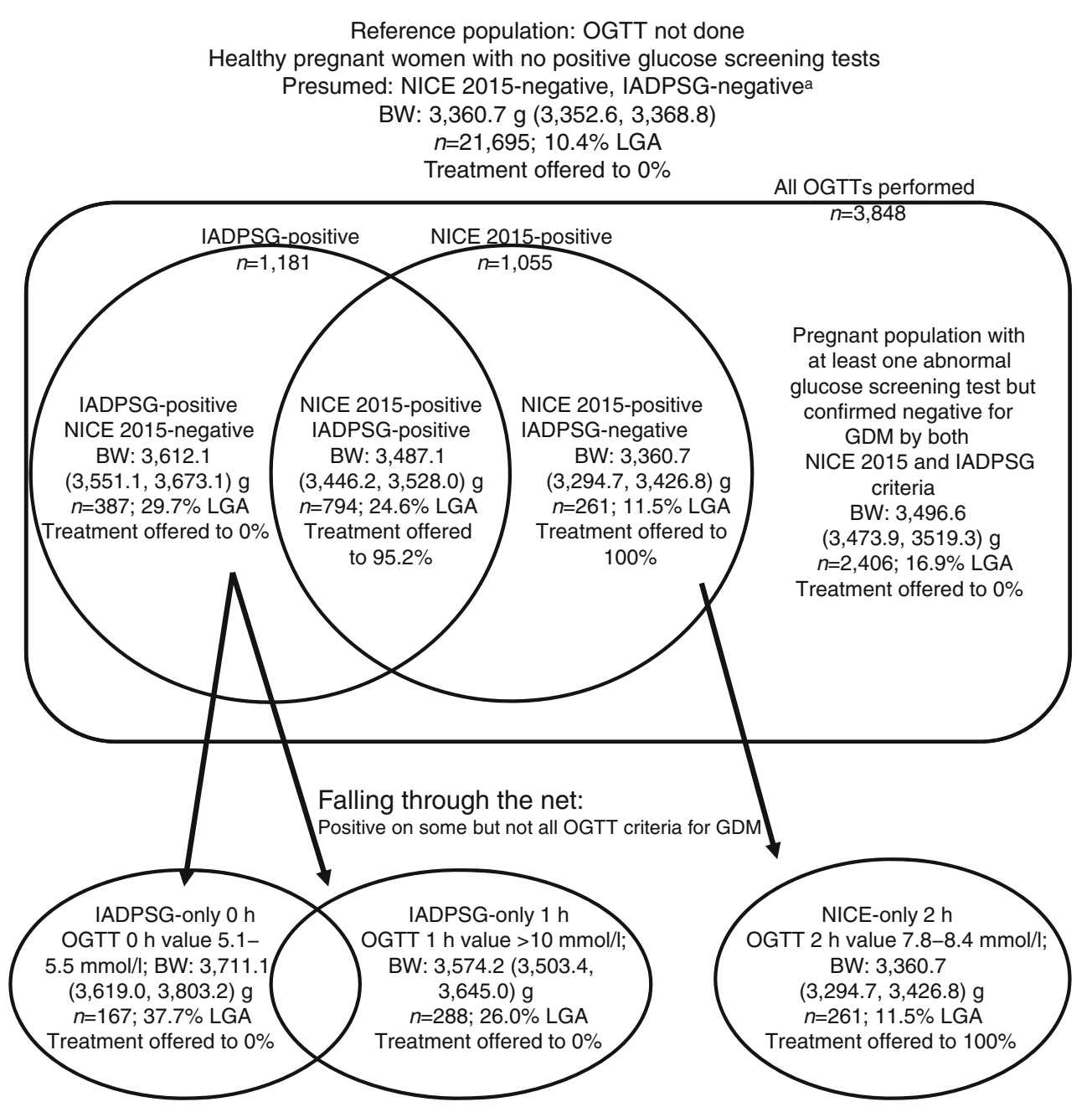

$4.13 \%(1,055 / 25,543)$, using NICE 2015 criteria, and 4.62\% $(1,181 / 25,543)$ according to the IADPSG criteria. Using the IADPSG criteria instead of the proposed NICE 2015 criteria would have resulted in treating 126 more women over 5 years. Although these 126 women represented only $0.49 \%$ of pregnancies, they accounted for $3.82 \%$ of cases of LGA, $2.68 \%$ of cases of pre-eclampsia and $5.30 \%$ of cases of polyhydramnios. Overall, 3,010 (12.2\%) infants had a birthweight above the 90th percentile, of whom 207 (6.9\%) mothers had been offered treatment for hyperglycaemia.

Characteristics of women with abnormal glucose tests As expected, women with GDM diagnosed by any method were older and had a higher BMI compared with the general population (Table 2). Pregnancies complicated by one or more abnormal glucose values yielded an infant with a higher birthweight (Tables 2, 3). Women who were offered treatment for GDM delivered infants with an average birthweight of 3 , $437 \mathrm{~g}$ and a higher rate of macrosomia (adjusted OR 1.49 $[1.21,1.84]$ ) and LGA (adjusted OR 1.84 [1.54, 2.20]) compared with the reference population, after adjustment for maternal age, parity, BMI, smoking status and ethnicity (and estimated gestational age at birth for macrosomia outcome). Women who had GDM by any criteria or both criteria were more likely to have a CS delivery and to suffer from preeclampsia compared with the reference population.

Pregnancies where an OGTT was performed that was negative for GDM according to the NICE and IADPSG criteria (NICE-negative IADPSG-negative) were at higher risk of macrosomia (16.8\%; unadjusted OR 1.60 [1.42, 1.79], adjusted OR 1.52 [1.34, 1.73]), LGA (16.9\%; unadjusted OR 1.75 [1.56, 1.96], adjusted OR $1.63[1.44,1.84])$, CS delivery (33.9\%; unadjusted OR $1.55[1.42,1.70]$, adjusted OR 1.36 [1.23, 1.51]) (especially emergency CS [19.7\%; unadjusted OR 1.45 (1.30, $1.61)$, adjusted OR $1.31(1.16,1.47)])$, pre-eclampsia (7.2\%; unadjusted OR $1.40[1.18,1.65]$, adjusted model did not show a significant effect) and polyhydramnios (4.4\%; unadjusted OR 8.15 [6.26, 10.61], adjusted OR $7.90[5.94,10.53])$ compared with the reference population. These women had abnormal glucose tests on screening and/or a history of previous GDM. 


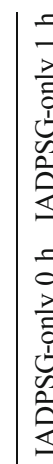

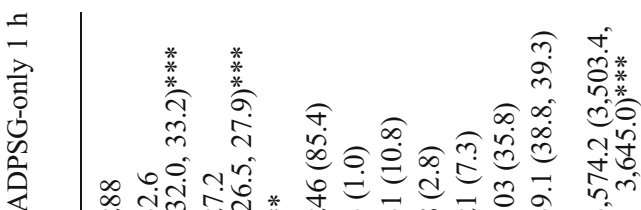

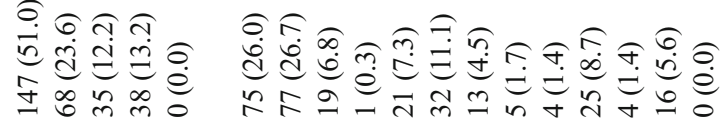

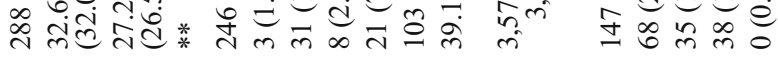

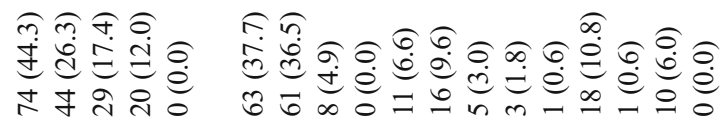

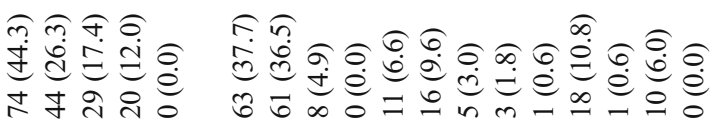

草

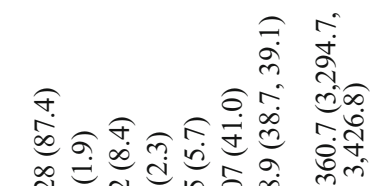

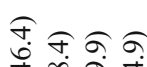

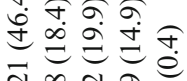

กิ่

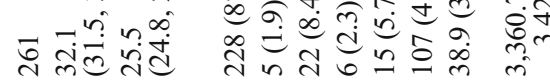

공 시

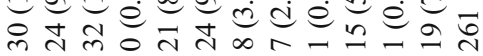

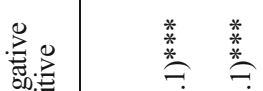

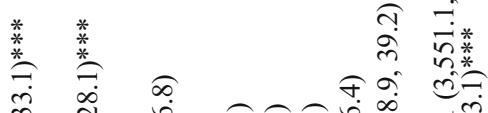

कितेंत्रं

สิติศ

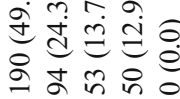

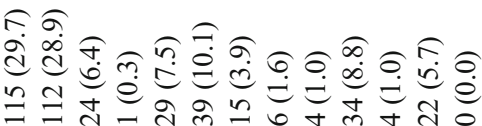

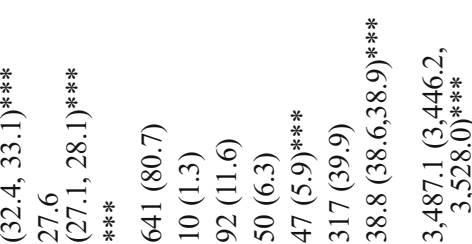

a. $\sigma=$

过 己ृ

6 ?

용 $\frac{n}{0}$

dُ

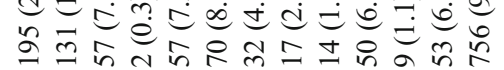

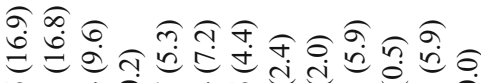

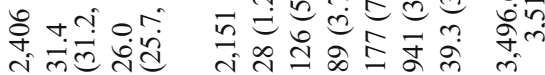

薄学志

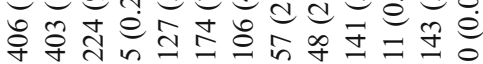

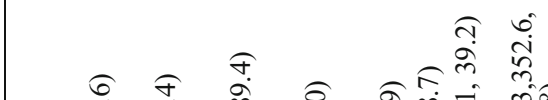

तె

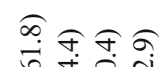

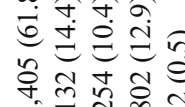

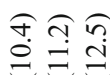

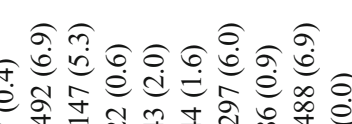

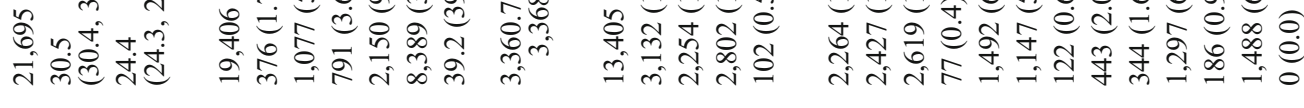

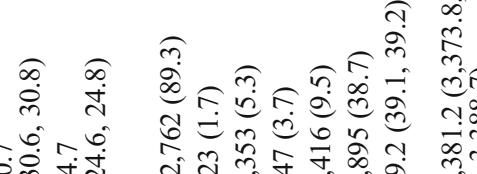

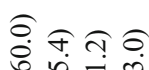

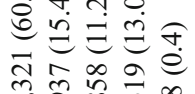

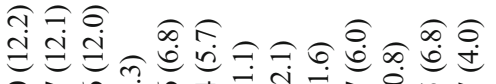

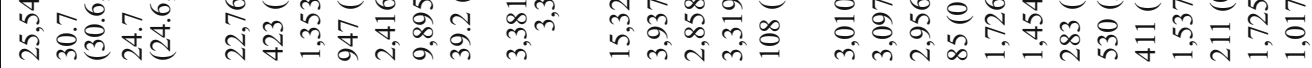

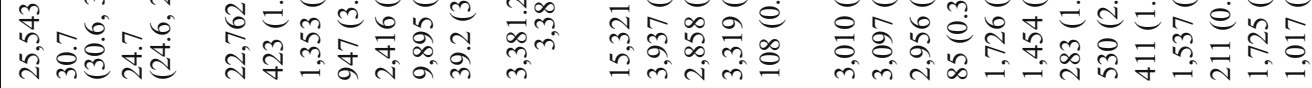
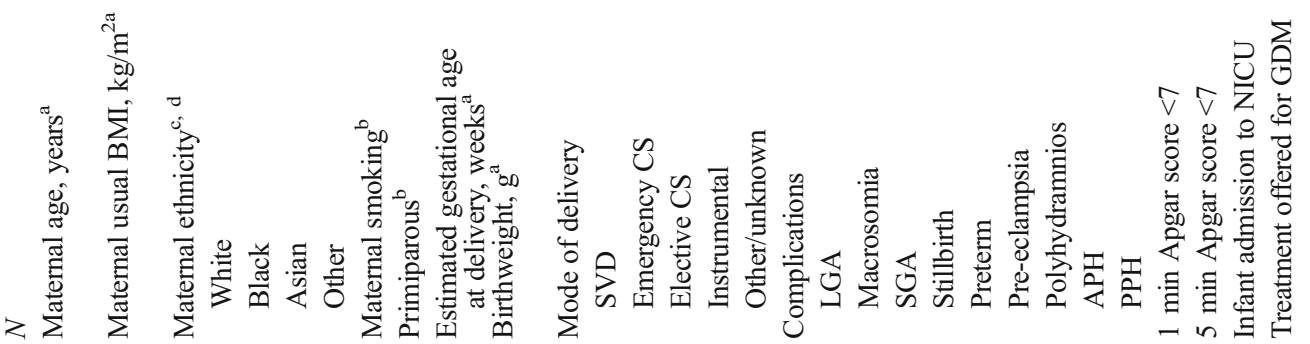

을 ㅇํㄴ 


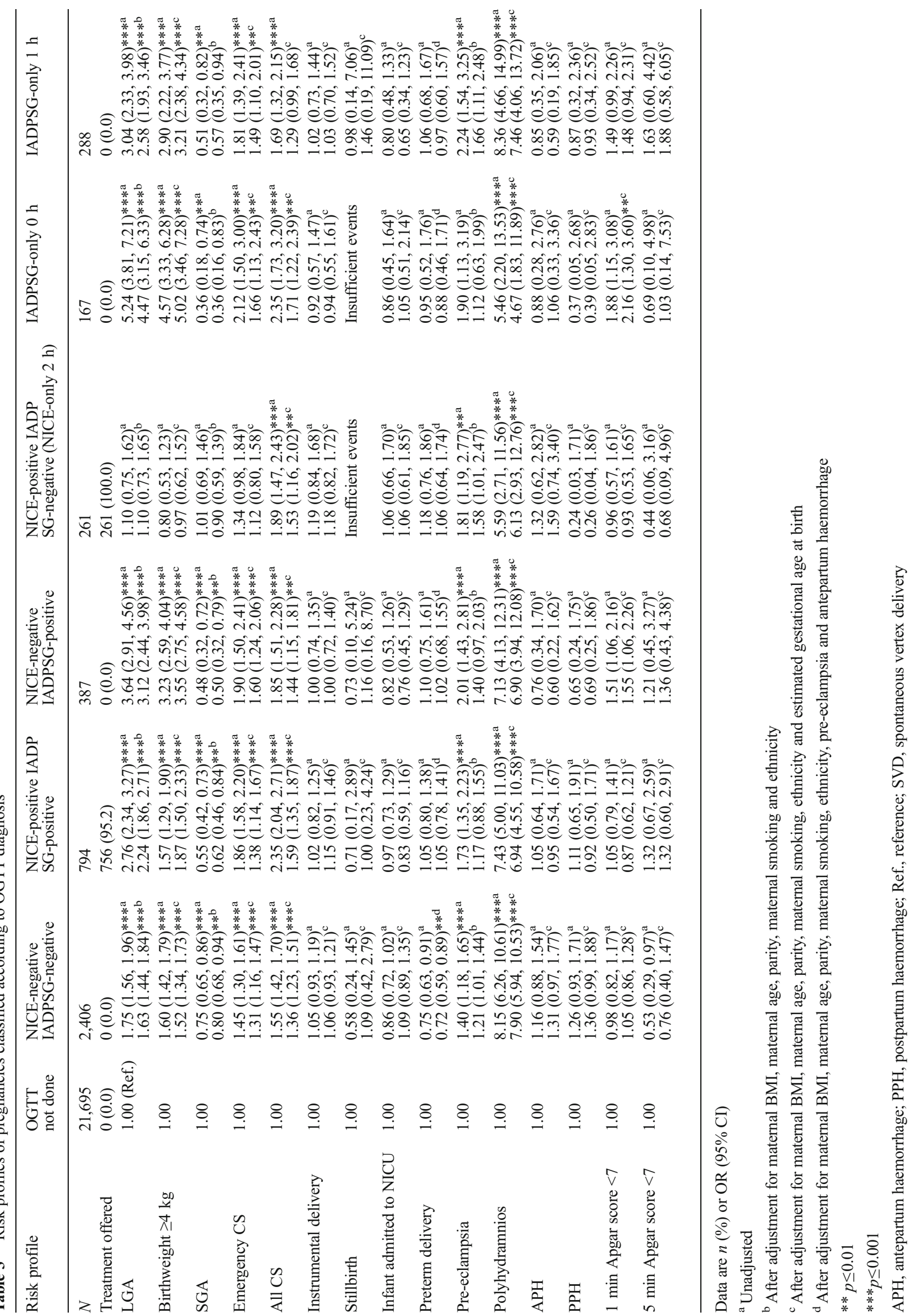


Women who were NICE-positive IADPSG-negative on OGTT were offered treatment and had an LGA rate comparable to that of the reference population (adjusted OR $1.10[0.73$, $1.65]$; $11.5 \%$ vs $10.4 \%$; Table 3 ). Overall, there was a trend for increased CS rates in this group (adjusted OR 1.53 [1.16, 2.02; $p=0.003]$ ), but there was no increase in emergency CS rates (adjusted OR $1.12[0.80,1.58]$ ). Pregnancies in this category were at increased risk of polyhydramnios (adjusted OR 6.13 $[2.93,12.76])$ but not of pre-eclampsia (adjusted OR 1.58 $[1.01,2.47])$ compared with the reference population.

Interestingly, women in the NICE-negative IADPSG-positive category who were untreated had the highest rate of LGA in this study (29.7\%; adjusted OR 3.12 [2.44, 3.98]). These women were at higher risk of emergency CS delivery (adjusted OR $1.60[1.24,2.06]$ ) and polyhydramnios (adjusted OR $6.90[3.94,12.08])$ compared with the reference population.

\section{Women who fall through the net with NICE 2015: identi-} fying the group at highest risk of LGA and other adverse outcomes Women in both the IADPSG-only $0 \mathrm{~h}$ and IADP SG-only $1 \mathrm{~h}$ groups were at high risk of LGA, CS delivery and emergency CS compared with the reference population, but the risks were higher for the IADPSG-only $0 \mathrm{~h}$ group: $37.7 \%$ and $26.0 \%$ of IADPSG-only $0 \mathrm{~h}$ and IADPSG-only $1 \mathrm{~h}$ pregnancies, respectively, yielded an LGA infant. Infants in the IADPSG-only $0 \mathrm{~h}$ group had a mean birthweight of 3,711.1 g: $350.4 \mathrm{~g}$ higher than infants from the reference population. They also had a trend for an increased risk of having a low 1 min Apgar score (adjusted OR 2.16 [1.30, 3.60]; $p=$ $0.003)$. Pregnancies in the IADPSG-only $1 \mathrm{~h}$ group had the greatest risk of polyhydramnios (adjusted OR 7.46 [4.06, 13.72]) and pre-eclampsia (unadjusted OR 2.24 [1.54, 3.25]) when compared with the reference population. However, when adjustment was made for maternal BMI, age, parity, smoking, ethnicity and estimated gestational age at birth, the risk of pre-eclampsia was no longer significant.

Overall, 20.4\% of women who were offered treatment for hyperglycaemia in pregnancy had an LGA infant. Although many of these women had more severe hyperglycaemia, this information was used to give a conservative estimate for the number needed to treat (NNT) to prevent one case of LGA in the groups which did not receive treatment. NNTs for the IADPSG-only $0 \mathrm{~h}$ and IADPSG-only $1 \mathrm{~h}$ groups were 5.8 and 17.9 , respectively.

\section{Discussion}

In this study, the prevalence of GDM in the study population was 4.13\% using NICE 2015 criteria and 4.62\% using IADP SG criteria. Using the IADPSG criteria instead of the proposed NICE 2015 criteria would have resulted in treating 126 more women over 5 years. Using the proposed NICE
2015 criteria would have resulted in $0.49 \%$ fewer women being offered treatment but would have failed to identify $3.82 \%$ cases of LGA, $2.68 \%$ cases of pre-eclampsia and $5.30 \%$ cases of polyhydramnios.

In this study, the women at highest risk of having an LGA infant were those who had an abnormal OGTT but 'fell through the net' between the IADPSG and NICE 2015 diagnostic cut-offs for GDM. Many of these women were not identified according to the diagnostic thresholds used at the time in our institution (75 g OGTT $0 \mathrm{~h} \geq 6.1 \mathrm{mmol} / \mathrm{l}$ or $\geq 7.1 \mathrm{mmol} / \mathrm{l} ; 2 \mathrm{~h} \geq 7.8 \mathrm{mmol} / \mathrm{l})$ and therefore were not offered treatment. Women with more severe degrees of hyperglycaemia were offered treatment and their offspring had a much lower risk of being LGA. Previous reports have suggested that offering treatment to women with modest degrees of hyperglycaemia in pregnancy results in a reduction in birthweight of 100-140 g [13]. However, in this study, women with an FPG 5.1-5.5 mmol/l (IADPSG-only $0 \mathrm{~h}$ ) gave birth to infants with a very high rate of LGA (37.7\%) and a mean birthweight $350 \mathrm{~g}$ higher than that of the reference population and $274 \mathrm{~g}$ higher than that of women with treated hyperglycaemia. A detailed cost-benefit analysis was beyond the scope of this project, but an NNT of 5.8 for this group suggests that treatment may be economical depending upon the expected risks in the population and compares favourably to other interventions in diabetes [22].

This study has several strengths. Data were collected on all singleton pregnancies at our institution between 2004 and 2008. This allows the assessment of outcomes and risk in a real-life clinical setting. Unlike many prospective clinical studies, the individuals were not highly selected and there was no difference between their care and standard clinical care at the time. However, this was a single centre study in a population with relatively low levels of ethnic diversity. Overall, the prevalence of GDM in our population was relatively low. Higher incidence rates for hyperglycaemia in pregnancy have been reported in other regions worldwide [23]. The IADPSG guidelines [11] now recommend screening pregnant women who are not known to have diabetes at 24-28 weeks' gestation using the OGTT and not the GCT. The use of the OGTT for universal screening of pregnant women may be associated with an increase in the prevalence of GDM in our population. However, the increased use of the OGTT also makes the issue of 'falling through the net' more important, as the number of women who test positive for the IADPSG and negative for the NICE 2015 criteria will be higher.

Using a retrospective study design confers some disadvantages. First, the screening protocol in use at the time used a GCT to exclude women who did not need an OGTT. Women with fasting hyperglycaemia, shown in this study to be at risk of adverse outcomes, would not have been readily identified using a GCT, which relies on a $1 \mathrm{~h}$ post-load test only. However, if these women had been removed from the analysis, the 
reference population would have been lower risk overall and the differences in outcome between reference and study populations would have been similar or more marked. Although screening tests were offered to all pregnant women, a minority might have chosen not to be screened, or not to attend for an OGTT if an initial screening test was positive. All women meeting predetermined criteria were offered dietary advice and other treatment, but response to treatment offered, the nature and dose of such treatments, and adherence to any advice or treatments received are unclear. Women with GDM received more antenatal scans and appointments and may be at greater risk of interventions or conditions such as pre-eclampsia and polyhydramnios through increased contact with health professionals. Although we cannot adequately control for this, it is interesting to note that women who were NICE-negative IADPSG-positive also had increased rates of pre-eclampsia and CS despite being considered not to have GDM at the time. Some capillary blood glucose samples were used in place of venous plasma for blood glucose measurement, which might have introduced small variations in measured glucose concentrations. A further consideration is that following an abnormal GCT result, some women might have instituted lifestyle change prior to OGTT testing, producing a better-than-expected OGTT result. While lifestyle change has been shown to improve glucose tolerance in obese pregnant women [24], the 1-2 week window between an abnormal GCT and a follow-up OGTT gives limited time for this, and further opportunities for GDM diagnosis were available later in pregnancy for women with symptoms or ultrasound features consistent with macrosomia. Information on neonatal sex was unavailable and therefore sex-specific criteria for LGA could not be used [25]. Although the sample size was large overall, the number of women in certain subgroups was too small to enable meaningful analysis of rare outcomes such as stillbirth. A $75 \mathrm{~g}$ OGTT was used, rather than the $100 \mathrm{~g}$ OGTT favoured by the American College of Obstetricians and Gynecologists (ACOG), which does not permit direct comparisons to be made with the ACOG diagnostic criteria. However, the ACOG criteria (100 g OGTT $0 \mathrm{~h} \geq 5.3 \mathrm{mmol} / \mathrm{l} ; 1 \mathrm{~h}$ $\geq 10.0 \mathrm{mmol} / \mathrm{l} ; 2 \mathrm{~h} \geq 8.6 \mathrm{mmol} / \mathrm{l} ; 3 \mathrm{~h} \geq 7.8 \mathrm{mmol} / \mathrm{l}$ ) will also identify many women with fasting hyperglycaemia, although as two abnormal results are required to make a diagnosis not all in this group would be offered treatment.

There is a fundamental difference between the IADPSG and NICE 2015 criteria. The IADPSG criteria are grounded on minimising the risk of harm to the mother and baby, and diagnostic thresholds were set to give an OR of 1.75 at each OGTT time point [11]. One of the challenges in ascertaining GDM-related risks in pregnancy is the large number of relatively infrequent adverse outcomes that have not shown consistent evidence of a significant association with GDM. For example, in the current study of 25,543 pregnancies, there were insufficient events to determine any effect of GDM classification on stillbirth rates or maternal admission to intensive care, and there was no evidence of altered rates of infant admission to the NICU. However, despite these concerns, the IADPSG criteria have been shown to be cost-effective, at least partly because of a reduction in the risk of infrequent adverse effects such as NICU admission [26]. Conversely, the NICE 2015 criteria have been based upon reducing historical average National Health Service unit costs for selected adverse outcomes (those common enough to have statistical power to be detected in randomised controlled studies) using health economic modelling. While cost-effectiveness is important in any healthcare system, the burden of psychological and emotional distress caused by many complications is also important and cannot be measured in economic terms alone. GDM diagnostic criteria should aim to identify women at risk of complications in the first instance, although not all these women may require pharmacological treatment. Future work should focus on accurate identification of women with GDM who are at low risk of complications and who might be suitable for community-based lifestyle interventions.

This study demonstrates a significant risk of LGA among infants of women who meet the IADPSG criteria for GDM who would be unidentified and untreated using many criteria in current clinical use (WHO 1999, modified WHO 1999 and proposed NICE 2015) [27]. One barrier to adoption of the IADPSG criteria for GDM has been the widespread concern about increased case identification rates and increased treatment rates leading to increased costs with purportedly little outcome data to support such a change [12]. Some reports have suggested that diagnosis rates of GDM would increase dramatically under the IADPSG criteria [13]. The current dataset suggests that adopting the IADPSG criteria over the NICE 2015 guidelines would be associated with a small increase in the prevalence of GDM from $4.13 \%$ to $4.62 \%$ in this population if screening protocols were unchanged (an additional 25.2 patients per year). Worryingly, these data suggest that if the NICE 2015 criteria were adopted instead of the IADPSG criteria a group of high-risk women would be unidentified and undertreated (IADPSG-only $0 \mathrm{~h}$ ) and a group of low-risk women (NICE-only $2 \mathrm{~h}$ ) would be treated instead. This suggests that despite being based upon cost-effectiveness, the NICE criteria may not necessarily facilitate the allocation of resources to address those most at risk of adverse outcomes.

The IADPSG criteria include women with an FPG 5.1$5.5 \mathrm{mmol} / 1$ and an OGTT $1 \mathrm{~h}$ glucose $\geq 10.0 \mathrm{mmol} / 1 \mathrm{who}$, untreated, were associated with a high risk of delivering LGA infants in this study. The IADPSG criteria exclude women who have an OGTT $2 \mathrm{~h}$ glucose $7.8-8.4 \mathrm{mmol} / \mathrm{l}$, who were found in this study to have a very low risk of LGA in their offspring (11.5\%). Although many in this group would have been offered treatment, a confounding factor, the low rate of LGA, suggests that some of these women could be safely 
untreated. Interestingly, these treated women had a lower incidence of LGA among their infants compared with untreated women who had an OGTT that was negative for GDM according to both NICE and IADPSG criteria despite having abnormal screening tests or previous GDM. The offspring of these women with a negative OGTT had a $16.9 \%$ risk of LGA, significantly higher than that of the reference population. These results confirm that women with borderline hyperglycaemia who have positive screening tests but who do not meet the diagnostic thresholds for GDM are also at increased risk of adverse outcomes. This finding suggests that standard dietary and lifestyle advice given in GDM might benefit even non-GDM women in pregnancy and could be given more widely.

One issue which has prevented widespread adoption of the IADPSG diagnostic criteria is the concern about unnecessarily over-medicalising healthy pregnancies [13]. In this study, even women with a negative OGTT were at higher risk of CS delivery compared with the reference population. The highest risks of CS delivery in this study were seen in women with a positive OGTT (NICE-positive IADPSG-positive) and in women with an FPG 5.1-5.5 mmol/1 (IADPSG-only $0 \mathrm{~h}$ ). Recommendations to offer induction at 38 weeks to women with treated GDM are likely to explain the increased rates of emergency CS in the NICE-positive IADPSG-positive group due to the risk of failed induction [17]. However, the IADP SG-only $0 \mathrm{~h}$ group had higher rates of emergency CS delivery overall, suggesting that women with unidentified hyperglycaemia had increased intervention rates despite being considered GDM-negative according to the diagnostic criteria in use in our institution at the time. These findings suggest that intervention rates may be related to glucose per se rather than diagnostic categorisation using the OGTT.

In summary, women who fall through the net, who would test positive for GDM according to the IADPSG criteria but not the NICE 2015 criteria, had the highest risk of having infants with LGA in this retrospective study compared with women in the reference population or those with more severe degrees of hyperglycaemia who were offered treatment. Women with FPG levels $5.1-5.5 \mathrm{mmol} / 1$ were at particularly high risk of CS delivery and LGA. Conversely, women with an OGTT 2 h glucose $7.8-8.4 \mathrm{mmol} / \mathrm{l}$, who would have had GDM according to the NICE 2015 criteria but not the IADP SG criteria, were offered treatment and had an extremely low incidence of LGA. These data demonstrate that the IADPSG criteria identify women at substantial risk of LGA who may benefit from treatment while excluding women who may have a low risk of adverse pregnancy outcomes.

Acknowledgements The authors acknowledge D. Church (Addenbrooke's Hospital, Cambridge, UK), E. Te Braake, K. Stubbington (The Rosie Hospital and Addenbrooke's Hospital, Cambridge, UK) and M. Wilson (Addenbrooke's Hospital, Cambridge, UK) for their contributions to data collection, and A. Thornton and A. Herrick (Addenbrooke's Hospital, Cambridge, UK) for their contributions to data extraction.

Funding This project was not supported by any specific funding.

Duality of interest CLM receives salary funding from the European Union Seventh Framework Programme (FP7/2007-2013; grant agreement number 266408) and from the Wellcome Trust Translational Medicine and Therapeutics Programme, which is funded by the Wellcome Trust in association with GlaxoSmithKline. HRM has received honoraria for speaking engagements from Medtronic, Roche, Novo Nordisk and Eli Lilly and is a member of the Medtronic European Advisory Board. DS has received honoraria from Sanofi and Novo Nordisk and is a member of the GlaxoSmithKline Advisory Panel. HBL and CP declare that there is no duality of interest associated with their contribution to this manuscript.

Contribution statement CLM identified the study question, designed the study, assisted with data analysis and interpretation of data, and wrote and revised the manuscript. HBL performed the statistical analysis and reviewed and edited the manuscript. $\mathrm{CP}$ made a substantial contribution to data acquisition and analysis, reviewed and revised the manuscript and contributed to the discussion. HRM made a substantial contribution to data acquisition and analysis, reviewed and revised the manuscript and contributed to the discussion. DS made a substantial contribution to data acquisition and analysis, reviewed and revised the manuscript and contributed to the discussion. All authors gave approval of the final version of the manuscript prior to publication. CLM is the guarantor of this work and, as such, had full access to all the data in the study and takes responsibility for the integrity of the data and the accuracy of the data analysis.

Open Access This article is distributed under the terms of the Creative Commons Attribution 4.0 International License (http:// creativecommons.org/licenses/by/4.0/), which permits unrestricted use, distribution, and reproduction in any medium, provided you give appropriate credit to the original author(s) and the source, provide a link to the Creative Commons license, and indicate if changes were made.

\section{References}

1. Metzger BE, Coustan DR (1998) Summary and recommendations of the Fourth International Workshop-Conference on Gestational Diabetes Mellitus. The Organizing Committee. Diabetes Care 21(Suppl 2):B161-B167

2. World Health Organisation (2013) Diagnostic criteria and classification of hyperglycaemia first detected in pregnancy. Available from http://apps.who.int/iris/bitstream/10665/85975/1/WHO NMH_MND_13.2_eng.pdf, accessed 20 Apr 2015

3. Simmons D (2010) Epidemiologic context of diabetes in pregnancy. In: McCance DR, Maresh M, Sacks DA (eds) A practical manual of diabetes in pregnancy. Blackwell, Oxford, pp 3-17

4. Stotland NE, Caughey AB, Breed EM, Escobar GJ (2004) Risk factors and obstetric complications associated with macrosomia. Int J Gynaecol Obstet 87:220-226

5. Ovesen PG, Jensen DM, Damm P, Rasmussen S, Kesmodel US (2015) Maternal and neonatal outcomes in pregnancies complicated by gestational diabetes. A nation-wide study. J Matern Fetal Neonatal Med. doi:10.3109/14767058.2014.966677

6. Ornoy A (2011) Prenatal origin of obesity and their complications: gestational diabetes, maternal overweight and the paradoxical effects of fetal growth restriction and macrosomia. Reprod Toxicol $32: 205-212$ 
7. Boney CM, Verma A, Tucker R, Vohr BR (2005) Metabolic syndrome in childhood: association with birth weight, maternal obesity, and gestational diabetes mellitus. Pediatrics 115:e290-e296

8. Crowther CA, Hiller JE, Moss JR et al (2005) Effect of treatment of gestational diabetes mellitus on pregnancy outcomes. N Engl J Med 352:2477-2486

9. Moss JR, Crowther CA, Hiller JE et al (2007) Costs and consequences of treatment for mild gestational diabetes mellitus - evaluation from the ACHOIS randomised trial. BMC Pregnancy Childbirth 7:27

10. Metzger BE, Lowe LP, Dyer AR et al (2008) Hyperglycemia and adverse pregnancy outcomes. N Engl J Med 358:1991-2002

11. Metzger BE, Gabbe SG, Persson B et al (2010) International Association of Diabetes and Pregnancy Study Groups recommendations on the diagnosis and classification of hyperglycemia in pregnancy. Diabetes Care 33:676-682

12. Vandorsten JP, Dodson WC, Espeland MA et al (2013) NIH consensus development conference: diagnosing gestational diabetes mellitus. NIH Consens State Sci Statements 29:1-31

13. Cundy T, Ackermann E, Ryan EA (2014) Gestational diabetes: new criteria may triple the prevalence but effect on outcomes is unclear. BMJ 348:g1567

14. American Diabetes Association (2014) Standards of medical care in diabetes-2014. Diabetes Care 37(Suppl 1):S14-S80

15. National Institute for Health and Care Excellence (NICE) (2015) Diabetes in pregnancy: management of diabetes and its complications from preconception to the postnatal period. Clinical guideline NG3 (2015). Available from www.nice.org.uk/guidance/ng3/ resources/diabetes-in-pregnancy-management-of-diabetes-and-itscomplications-from-preconception-to-the-postnatal-period51038446021, accessed 20 Apr 2015

16. Church D, Halsall D, Meek C, Parker RA, Murphy HR, Simmons D (2011) Random blood glucose measurement at antenatal booking to screen for overt diabetes in pregnancy: a retrospective study. Diabetes Care 34:2217-2219

17. National Institute for Health and Care Excellence (NICE) (2008) Diabetes in pregnancy: management of diabetes and its complications from preconception to the postnatal period. Clinical guideline
63 (2008). Available from www.nice.org.uk/guidance/cg63/ resources/guidance-diabetes-in-pregnancy-management-ofdiabetes-and-its-complications-from-preconception-to-thepostnatal-period-pdf, accessed 16 May 2015

18. Kupke IR, Kather B, Zeugner S (1981) On the composition of capillary and venous blood serum. Clin Chim Acta 112:177-185

19. Hadlock FP, Harrist RB, Martinez-Poyer J (1991) In utero analysis of fetal growth: a sonographic weight standard. Radiology 181: $129-133$

20. Mikolajczyk RT, Zhang J, Betran AP et al (2011) A global reference for fetal-weight and birthweight percentiles. Lancet 377:1855-1861

21. Gardosi J, Mongelli M, Wilcox M, Chang A (1995) An adjustable fetal weight standard. Ultrasound Obstet Gynecol 6:168-174

22. American Diabetes Association and the National Institute of Diabetes and Digestive and Kidney Diseases (2004) Prevention or delay of type 2 diabetes. A position statement. Diabetes Care 27(Suppl 1):S37-S54

23. Guariguata L, Linnenkamp U, Beagley J, Whiting DR, Cho NH (2014) Global estimates of the prevalence of hyperglycaemia in pregnancy. Diabetes Res Clin Pract 103:176-185

24. Vinter C, Jørgensen JS, Ovesen P, Beck-Nielsen H, Skytthe A, Jensen DM (2014) Metabolic effects of lifestyle intervention in obese pregnant women. Results from the randomized controlled trial 'Lifestyle in pregnancy' (LiP). Diabetes Med 31:1323-1330

25. Gardosi J, Chang A, Kalyan B, Sahota D, Symonds EM (1992) Customised antenatal growth charts. Lancet 339:283-287

26. Duran A, Sáenz S, Torrejón MJ et al (2014) Introduction of IADPSG criteria for the screening and diagnosis of gestational diabetes mellitus results in improved pregnancy outcomes at a lower cost in a large cohort of pregnant women: the st. Carlos Gestational Diabetes Study. Diabetes Care 37: $2442-2450$

27. National Health Service. Gestational diabetes mellitus: screening, diagnosis and follow-up. Available from www.diabetes.org.uk/ Documents/nhs-diabetes/pregnancy-preconception/gestationaldiabetes-screening-diagnosis-followup-national-overview.pdf, accessed 20 Apr 2015 